\title{
SIGNATURES OF EXOSOLAR PLANETS IN DUST DEBRIS DISKS
}

\author{
Leonid M. Ozernoy, ${ }^{1,2}$ Nick N. Gorkavyi, ${ }^{2}$ John C. Mather, ${ }^{2}$ and Tanya A. Taidakova ${ }^{3}$ \\ Received 1999 August 25; accepted 2000 May 31; published 2000 June 30
}

\begin{abstract}
We apply our recently elaborated, powerful numerical approach to the high-resolution modeling of the structure and emission of circumstellar dust disks, incorporating all relevant physical processes. Specifically, we examine the resonant structure of a dusty disk induced by the presence of one planet. It is shown that the planet, via resonances and gravitational scattering, produces (1) an asymmetric resonant dust belt with one or more clumps, intermittent with one or a few off-center cavities, and (2) a central cavity void of dust. These features can serve as indicators of a planet embedded in the circumstellar dust disk and, moreover, can be used to determine its major orbital parameters and even the mass of the planet. The results of our study reveal a remarkable similarity with various types of highly asymmetric circumstellar disks observed with the James Clerk Maxwell Telescope around $\epsilon$ Eridani and Vega. The proposed interpretation of the clumps in those disks as being resonant patterns is testable - it predicts the asymmetric design around the star to revolve, viz., by $1.2-1.6 \mathrm{yr}^{-1}$ about Vega and $0.6-0.8 \mathrm{yr}^{-1}$ about $\epsilon$ Eri.
\end{abstract}

Subject headings: circumstellar matter — dust, extinction — planetary systems stars: individual (Vega, $\epsilon$ Eridani)

\section{INTRODUCTION}

A significant limitation to unambiguous planet detection in circumstellar disks is the contaminating thermal emission from dust in the target systems. We propose to turn this hazard into an advantage for detecting and characterizing planetary systems. The major goal of the present Letter is to explore how the presence of the planet(s) impacts the disk via particular resonances that are responsible for the specific asymmetric features in the dust and how these features would enable us to derive the major parameters of the yet invisible planetary system. The major obstacle in providing reliable, high-resolution numerical simulations - the particle-number limitation - is solved by our recently elaborated and very efficient approach to numerical modeling of distributions of test particles in an external gravitational field (Ozernoy, Gorkavyi, \& Taidakova 1998, 2000; Gorkavyi et al. 2000; Taidakova \& Gorkavyi 1999).

\section{RESONANCE STRUCTURES IN DUSTY CIRCUMSTELLAR DISKS}

Both the Poynting-Robertson (P-R) and stellar wind drags tend to induce dust inflow toward the star. As the dust passes by the planets in its infall, it interacts with them by accumulating in the outer planetary resonances, which, as we demonstrate below, could serve as an efficient means of planet detection. The existence of a resonance ring associated with the Earth's orbit was predicted by Jackson \& Zook (1989); such a ring was indeed discovered in the IRAS (Dermott et al. 1994) and COBE (Reach et al. 1995) data. The resonant design in the dust depends on the capture probability, the particle lifetime in the resonance, the amplitude of libration, and the typical eccentricity of resonant particles.

The total mass of dust contained in a particular resonance

\footnotetext{
${ }^{1}$ Institute for Computational Sciences and Informatics, 5C3, and Department of Physics and Astronomy, 4400 University Drive, George Mason University, Fairfax, VA 22030-4444; ozernoy@ science.gmu.edu.

${ }^{2}$ NRC/NAS, Code 685, Laboratory for Astronomy and Solar Physics, NASA Goddard Space Flight Center, Greenbelt, MD 20771; ozernoy@stars.gsfc .nasa.gov, gorkavyi@stars.gsfc.nasa.gov, john.c.mather@gsfc.nasa.gov.

${ }^{3}$ Computational Consulting Services, College Park, MD 20740; simeiz@ aol.com.
}

is the product of the probability of the dust particle being captured into the resonance $W_{j}$, the lifetime spent in the resonance $\tau_{j}$ (in a sufficiently dense environment, this time is due to particle collisions), and the total dust flow per unit time in the vicinity of the resonance $d M / d t$, i.e., $M_{j} \approx W_{j} \tau_{j}(d M / d t)$. Having introduced, for convenience, the mass of "background" dust $M_{b} \approx\left(1-W_{j}\right) \tau(d M / d t)$ one gets $M_{j} / M_{b} \approx W_{j}\left(1-W_{j}\right)^{-1}\left(\tau_{j} / \tau\right)$, where $\tau$ is the minimal timescale of the P-R drag $\left(\tau_{\mathrm{PR}}\right)$, gravitational scattering $\left(\tau_{\mathrm{gs}}\right)$, and collisional $\left(\tau_{\text {coll }}\right)$ characteristic times. Three particular cases are well distinguishable:

1. $\tau \approx \tau_{\mathrm{PR}}<\tau_{\mathrm{gs}}, \tau_{\text {coll }}$. Actually, this collisionless case is realized, for example, for Neptune in the solar system. Assuming that $W_{j} \sim 1-W_{j}$, one gets $M_{j} / M_{b} \sim \tau_{j} / \tau_{\mathrm{PR}}$. To get a discernable resonant structure, one needs to have $\tau_{j} \sim \tau_{\mathrm{PR}}$.

2. $\tau \approx \tau_{\mathrm{gs}}<\tau_{\mathrm{coll}}, \tau_{\mathrm{PR}}$. This case is realized for a rather massive planet like Jupiter. If collisions are essential (as they are for Vega's disk; see Backman 1998), then $\tau_{j} \approx \tau_{\text {coll }}$, and one gets $M_{j} / M_{b} \sim \tau_{\text {coll }} / \tau_{\mathrm{gs}} \gtrsim 1$. The resonant structure has a high contrast if $\tau_{\mathrm{gs}} \ll \tau_{\text {coll }}$.

3. $\tau \approx \tau_{\text {coll }}<\tau_{\mathrm{gs}}, \tau_{\mathrm{PR}}$. In this collisional case, one has $\tau_{j} \approx$ $\tau_{\text {coll }}$, giving $M_{j} / M_{b} \sim W_{j} /\left(1-W_{j}\right)$, which results in a highcontrast resonant structure whenever $W_{j} \sim 1$.

It is worth emphasizing that if collisions of resonant particles are important (cases 2 and 3), the resonant pattern can nevertheless exist (cf. Wyatt et al. 1999) as long as a source of particle replenishment is available.

\section{MODELING CIRCUMSTELLAR DUST DISKS}

\subsection{The Procedure}

The simplest planetary system under consideration, consisting of just one planet (orbiting a star of mass $M$ at a circular orbit of radius $a$ ), possesses only mean motion resonances, without most of the secular resonances (except the Kozai resonances). We have computed about 300 model disks in order to explore the effects of various resonances, adopting the following parameters: (1) the mass of the planet $m_{\mathrm{pl}} ;$ (2) the particle size coupled with stellar luminosity and mass, as described by the parameter $\beta \approx 0.3 L_{*} /\left(M_{*} r\right)$, which is the ratio of the 
stellar light pressure and the gravitational force applied to a dust grain of density $2 \mathrm{~g} \mathrm{~cm}^{-3}$ and radius $r$ (in microns), with $L_{*}$ and $M_{*}$ as the star's luminosity and mass, respectively, in solar units; and (3) the lifetime of dust particles $\tau$. Below, we describe some of these models.

\subsection{Typical Resonant Structures Exemplified by the Resonances $1: 1,2: 1,3: 1$, and $3: 2$}

In the absence of a planet, the surface density of dust between the star and the pericenter of dust sources is constant, while the number density of dust varies as $r^{-1}$, with $r$ being the heliocentric distance (Gorkavyi et al. 1997). The presence of a planet induces dramatic changes in the dust distribution. This is illustrated in Figure 1, which shows the geometry of the dust distribution in different mean motion resonances with the planet.

In the resonance $1: 1$, the orbits responsible for the dust design are of three kinds: (1) a horseshoe-like orbit that embraces the two Lagrangian points L4 and L5 (see Fig. 1a), (2) an orbit with a small libration around L4 (Fig. 1b, upper feature), and (3) an orbit with a substantial libration around L5 (Fig. 1b, lower feature).

The dust structure in the resonance $2: 1$ looks similar: the inner orbital loop precesses relative to L4 and L5 in the same way as the epicycle of particles in the $1: 1$ resonance. That loop can form a horseshoe-like arc along the planet's orbit (Fig. 1c) or can librate near either L4 or L5 (Fig. $1 d$ illustrates the case of a large libration).

In the resonance $3: 1$, the dust particles librate around the L4 with a smaller amplitude (Fig. 1e); no horseshoe-like libration has been observed. Finally, Figure $1 f$ illustrates the resonance $3: 2$ with an appreciable libration, which results in a symmetric four-clump pattern. When libration is small, the resonance contains a two-clump pattern.

In all the cases mentioned above, the vicinity of the planet is free of dust. Each resonant dust structure is accompanied by other specific caverns in dust distribution (see Fig. 1).

\subsection{Dependence of Structure on Location of Dust Sources}

The scattered cometary population produces dust whose distribution in orbital elements tends to be rather smooth. A wellpronounced resonant structure in the dust can only be formed if there is a planet that is massive enough to capture some dust particles into resonances and to eject the remaining nonresonant dust particles out (case 2 in $\S 2$ ).

Dust sources like the Kuiper Belt objects (KBOs; hereafter "Kuiperoids"), of which a substantial part is in resonances, can produce the contrast resonant structures if (1) the drifting dust particles have a long lifetime and eventually are captured into resonances (the situation is similar to the formation of a resonant dust belt near the Neptunian orbit in the solar system) or (2) both $\beta$ and the lifetime of dust particles are small enough so that the particles produced by the resonant Kuiperoids cannot leave the resonances, thereby forming resonant structures (case 3 in $\S 2$ ). Orbits of resonant objects look similar to the dust orbits shown in Figure 1.

\subsection{Dependence of Structure on Planetary Mass}

This dependence can be revealed from a variety of available runs with different parameters. A low-mass planet, like Earth or Neptune, forms a resonant ring along the planetary orbit that is associated with high-order resonances like $3: 2,4: 3$, etc. (see, e.g., Fig. 1f). For more massive planets, the resonances like $2: 1,3: 1$, etc., become essential, whereas the innermost resonances overlap (e.g., Wisdom 1980). Since the libration amplitude decreases as $m_{\mathrm{pl}}$ increases, clumps similar to those seen in Figure 1e (and not arcs like those shown in Fig. 1d) would be typical for $m_{\mathrm{pl}}$ as large as $m_{\mathrm{J}}$, which is Jupiter's mass.

\section{A LINK TO OBSERVATIONS: IMAGERY OF CIRCUMSTELLAR DISKS}

Let us consider some observational signatures of planets embedded in disks. Figures $2 a$ and $2 b$ show the thermal emission from the simulated disks (seen face-on) with an embedded planet, and these are to be compared with the available observational data on Vega and $\epsilon$ Eri shown in Figures $2 c$ and $2 d$, respectively (the parent stars are seen almost pole-on).

As a hotter star, Vega might result in more extensive, compared with $\epsilon$ Eri, dust production from evaporating comets. We adopt $\beta=0.3$ (close to an upper limit of $\beta=0.5$ at which the dust particle escapes) and $\tau_{\text {coll }}=2 \times 10^{4}$ planet revolutions, which is consistent with $\tau_{\text {coll }} \sim 10^{7} \mathrm{yr}$ (Backman 1998). A planet as massive as $\sim 2 m_{\mathrm{J}}$ induces the resonances $n: 1$, which results in two dusty clumps on either side of the planet, while the planet ejects the remaining nonresonant dust particles out (case 2 in $\S 2$ ). Thus, even if the distribution of the cometary population around Vega might be symmetric, the ejection of nonresonant particles by the planet could make the resonant dust pattern highly asymmetric (Fig. $2 a$ ), which is reminiscent of the two asymmetric clumps observed near Vega shown in Figure $2 c$ (Holland et al. 1998).

In contrast to Vega, $\epsilon$ Eri is a less massive and colder star with $L \approx 1 / 180 L_{\mathrm{Vega}}$. Besides, the typical size of dust particles is several times larger than in Vega's disk (Backman 1998); therefore, we adopt $\beta=0.002$ for the dust in $\epsilon$ Eri's disk. Assuming that Kuiperoids serve as the main source of dust in this disk, we find that the dust distribution might reflect the distribution of the sources located mostly in resonances such as $2: 1$ and $3: 2$. In this case, a comparatively small planetary mass of $\sim 0.2 m_{\mathrm{J}}$ is able to induce, via libration, two arcs, with a pair of clumps at their edges (Fig. 2b). Even if the dust is distributed in equal proportions to both resonances, one could easily get the asymmetry seen in Figure $2 b$ by assuming that $10 \%$ of the comets in the resonance $2: 1$ have a smaller libration amplitude in the lower branch of the pattern. A similar asymmetric structure has been revealed in the submillimeter imagery of the $\epsilon$ Eri disk shown in Figure $2 d$ (Greaves et al. 1998).

Our modeling, which indicates that Vega may have a planet of mass $\sim 2 m_{\mathrm{J}}$ at a distance of 50-60 AU and that $\epsilon$ Eri may have a less massive planet of $m \approx 0.2 m_{\mathrm{J}}$ at a similar distance of 55-65 AU, is testable. Each resonant feature is stationary in the reference frame corotating with the planet, but it is not so for the observer at Earth. Therefore, if our interpretation of asymmetric clumps as a dynamical resonant structure is correct, the above asymmetric feature revolves around the star (probably counterclockwise) with an angular velocity of $1.2-1.6 \mathrm{yr}^{-1}$ (Vega) and $0.6-0.8 \mathrm{yr}^{-1}(\epsilon$ Eri) - a prediction that can be tested within several years. If confirmed, the proposed interpretation of the structure in Vega- and $\epsilon$ Eri-like circumstellar disks seen face-on would make it possible not just to reveal the embedded planet and determine its semimajor axis but also to constrain its other basic parameters, such as the planet's mass, and even to pinpoint the position of the planet (see Figs. $2 a$ and $2 b$ ). 


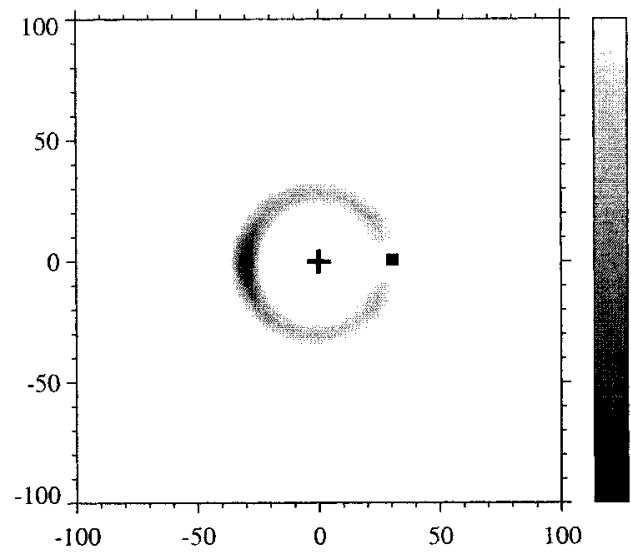

1a)

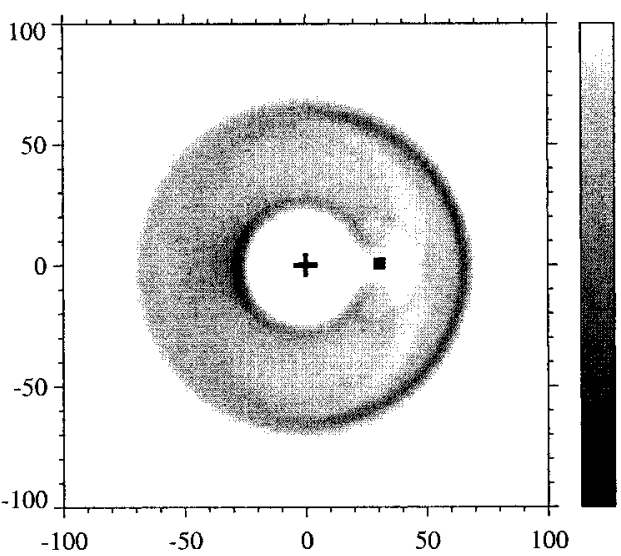

1c)

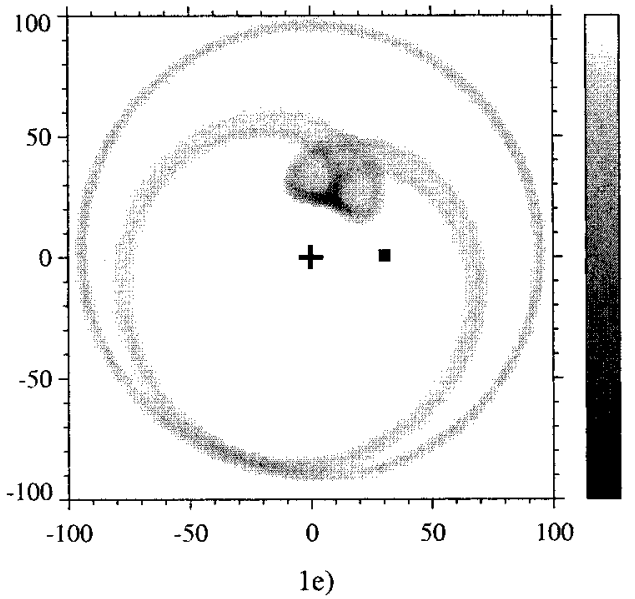

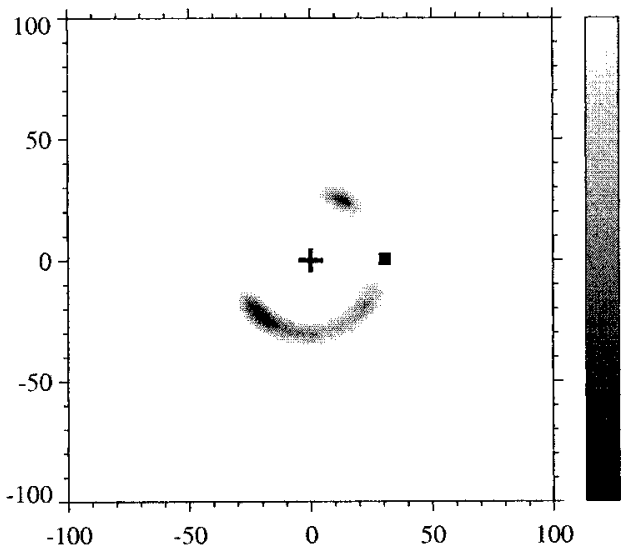

1b)

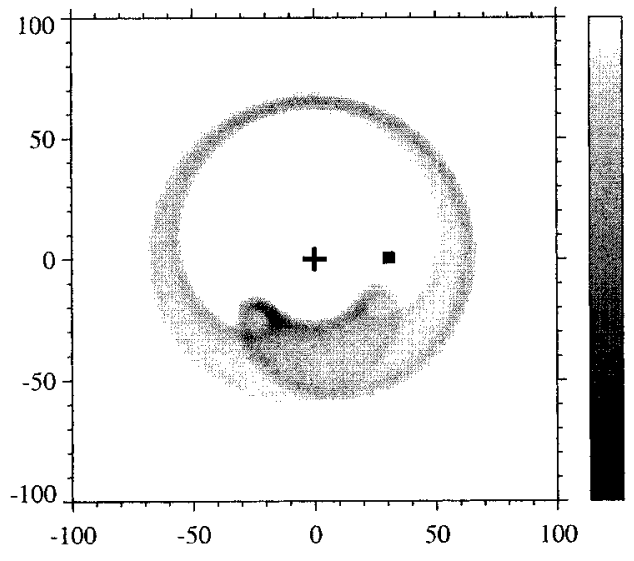

1d)

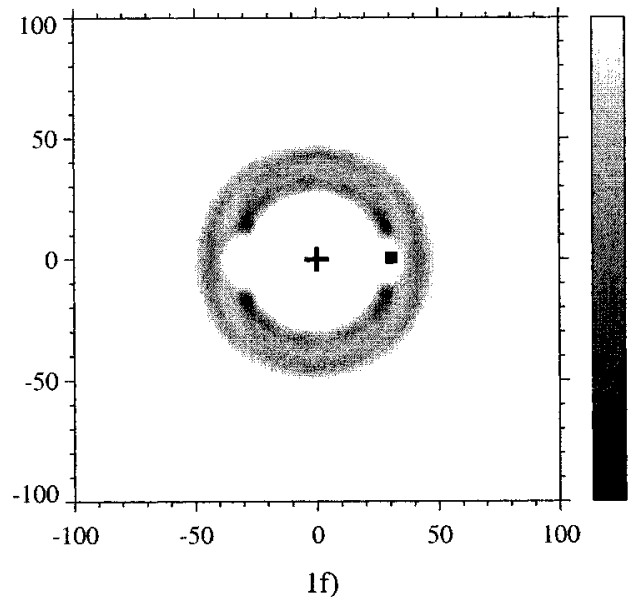

FIG. 1.-Simulated surface density of the circumstellar dust $(\beta=0.065)$ captured into particular mean motion resonances with an outer planet shown as a square. The star's location is $(0,0)$. In each panel, the surface density of the disk is shown on a linear contrast scale, the area covered is $200 \times 200$ (the scale is arbitrary, but for the solar system it would be in astronomical units), and the (single) planet is assumed to be on a circular orbit $\left(e_{\mathrm{pl}}=0\right)$ at $r=30$. The particle lifetime in the resonance $\tau_{\text {res }}$ was taken to be $10^{3}$ planet revolutions everywhere: $(a) m=0.3 m_{\mathrm{J}}$, resonance $1: 1$ (a horseshoe-like orbit around the two Lagrangian points L4 and L5); (b) same as (a), but with an orbit around L4 with a minimal libration and another, nonoverlapping orbit around L5 with a maximum libration; (c) same as $(a)$, but for resonance $2: 1 ;(d) m=0.3 m_{\mathrm{J}}$, resonance $2: 1$ (an orbit around L4 or L5 with a maximum libration); $(e) m=3 m_{\mathrm{J}}$, resonance $3: 1$ (an orbit around the Lagrangian point L4 with a smaller libration amplitude caused by a larger $m_{\mathrm{pl}}$ ). Note that at $m_{\mathrm{pl}}$ as small as $m=0.3 m_{\mathrm{J}}$, there is no capture into this resonance. $(f) m=0.3 m_{\mathrm{J}}$, resonance $3: 2$. 


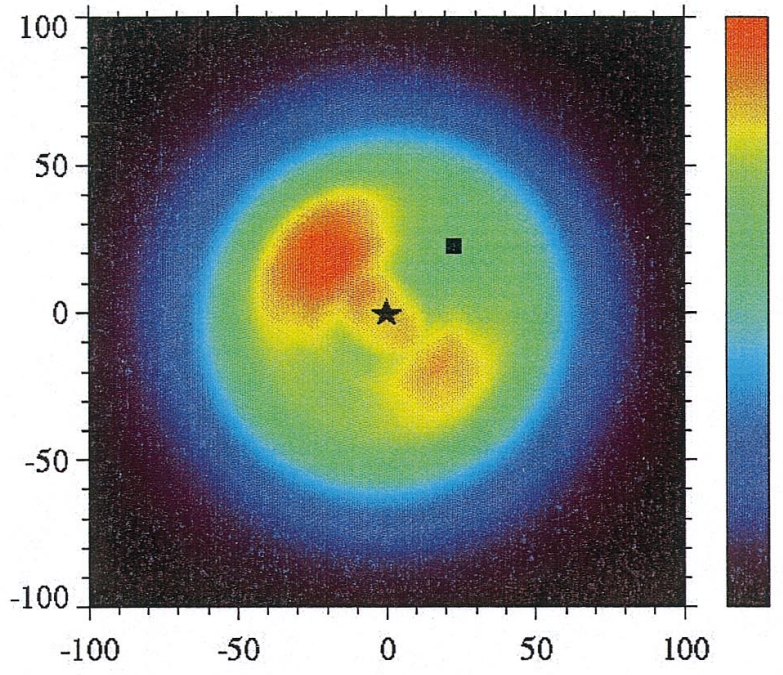

2a)

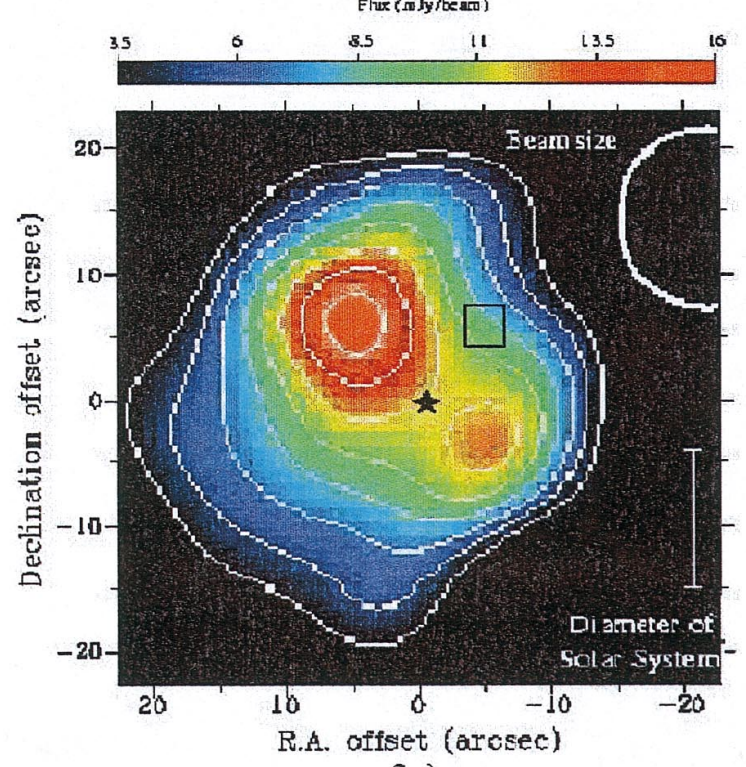

2c)
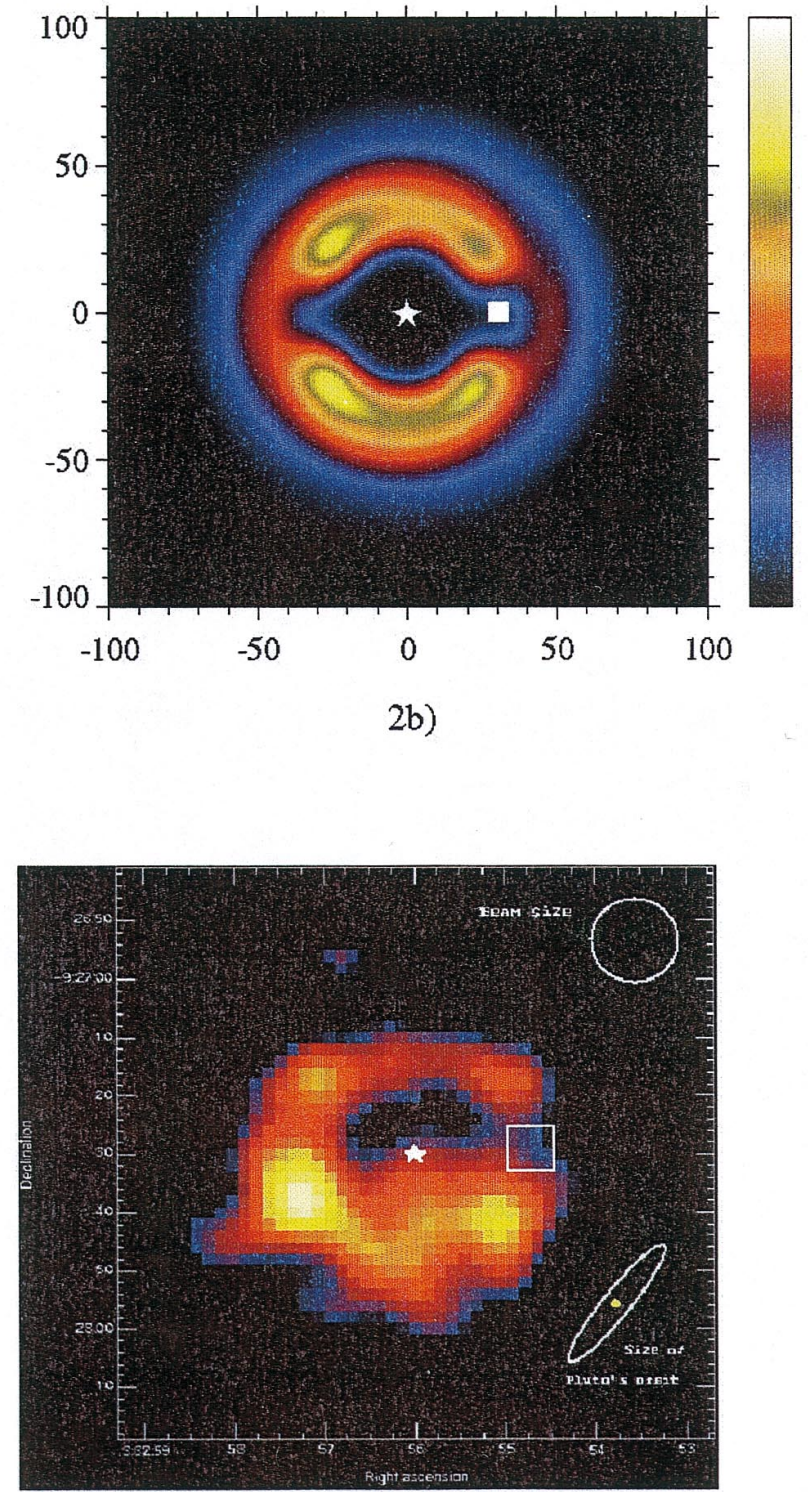

2d)

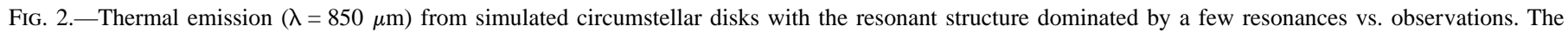

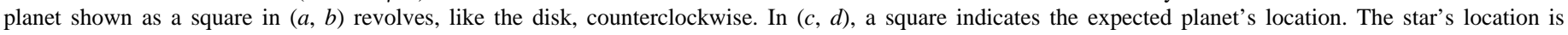

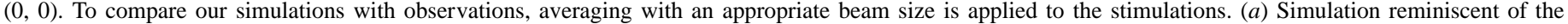

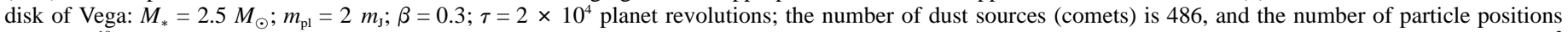

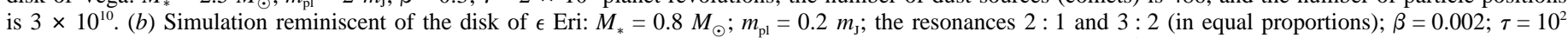

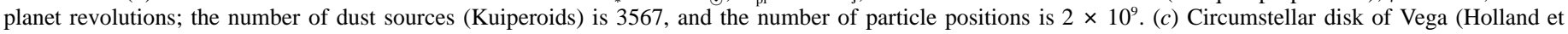
al. 1998). (d) Circumstellar disk of $\epsilon$ Eri (Greaves et al. 1998).

\section{CONCLUSIONS}

As we have demonstrated above, highly asymmetric structures in the circumstellar disk and a central "hole" or void of dust can serve as indicators of at least one planet embedded in the disk. The numerical simulations described above lead to the following major conclusions:

1. The outermost planet in an exoplanetary system can produce (a) an asymmetric resonant dust belt consisting of $\operatorname{arc}(\mathrm{s})$ and one or more clumps that are intermittent with one or a few cavities and $(b)$ a central cavity void of dust.

2. The morphology of these belts and cavities (size, asymmetry, number of clumps, and their pattern) depends on the particular resonances involved and is eventually a function of the planetary mass, the location of dust sources, and $\beta$.

3 . The crucial test of the above picture would be the discovery of the revolution of the resonant asymmetric structure around the star. For circumstellar disks in Vega and $\epsilon$ Eri, the asymmetric design is expected to revolve, respectively, by $1.2-1.6$ and $0.6-0.8$ annually.

In sum, our numerical simulations offer the possibility of revealing the presence of the planet(s) in a circumstellar dusty disk seen pole-on and, under further development, of providing a method for the determination of planetary parameters using the visible morphology of an outer part of the disk. The pro- 
posed approach seems to be sufficiently powerful so as to provide the interpretation of disks seen edge-on in scattered light, such as the $\beta$ Pic disk revealed by STIS optical observations (Heap et al. 2000).

This work has been supported by NASA grant NAG5-7065 to George Mason University. N. N. G. has been supported through the NAS/NRC Associateship Research program. The authors are thankful to Sally Heap for her help with programming and for her helpful discussions. We also thank W. S. Holland, J. S. Greaves, and their teams for permission to reproduce their SCUBA images.

\section{REFERENCES}

Backman, D. E. 1998, in Exozodiacal Dust Workshop, ed. D. E. Backman et al. (NASA CP-10155; Moffett Field: NASA/ARC), 13

Dermott, S. F., Jayaraman, S., Xu, Y. L., Gustafson, B. A. S., \& Liou, J. C. 1994, Nature, 369, 719

Gorkavyi, N. N., Ozernoy, L. M., Mather, J. C., \& Taidakova, T. 1997, ApJ, 488,268

- 2000, in ASP Conf. Ser. 207, NGST Science and Technology Expostion, ed. E. P. Smith \& K. S. Long (San Francisco: ASP), 462

Greaves, J. S., et al. 1998, ApJ, 506, L133

Heap, S. R., et al. 2000, ApJ, in press

Holland, W. S., et al. 1998, Nature, 329, 788
Jackson, A. A., \& Zook, H. A. 1989, Nature, 337, 629

Ozernoy, L. M., Gorkavyi, N. N., \& Taidakova,T. 1998, preprint (astro-ph/ 9812479)

. 2000, Planet. Space Sci., in press

Reach, W. T., et al. 1995, Nature, 374, 521

Taidakova, T. A., \& Gorkavyi, N. N. 1999, in The Dynamics of Small Bodies in the Solar System: A Major Key to Solar System Studies, ed. B. A. Steves, A. E. Roy, \& V. G. Szebehely (NATO ASI Ser. C, 522; Boston: Kluwer), 393

Wisdom, J. 1980, AJ, 85, 1122

Wyatt, M. C., et al. 1999, ApJ, 527, 918 